\title{
Fault-Tolerant Support for Reliable Multicast in Mobile Wireless Systems
}

\author{
Giuseppe Anastasi ${ }^{1}$, Alberto Bartoli ${ }^{2}$, and Flaminia L. Luccio $^{3}$ \\ 1 Dip. di Ingegneria dell'Informazione, Università di Pisa, Italy \\ anastasi@iet.unipi.it \\ 2 Dip. di Elettrotecnica, Elettronica e Informatica Università di Trieste, Italy \\ bartolia@univ.trieste.it \\ 3 Dip. di Scienze Matematiche, Università di Trieste, Italy \\ luccio@dsm.univ.trieste.it
}

\begin{abstract}
In this paper we present a protocol for reliable multicast within a group of mobile hosts that communicate with a wired infrastructure by means of wireless technology. The protocol tolerates failures in the wired infrastructure, i.e., crashes of stationary hosts and partitions of wired links. The wireless coverage may be incomplete and message losses could occur even within cells, due to physical obstructions or to the high error rate of the wireless technology, for example. Movements of mobile hosts are accommodated efficiently because they do not trigger any interaction among stationary hosts (i.e., there is no notion of handoff). We evaluate by simulation the impact of fault-tolerance on the performance of the protocol in normal operating conditions, i.e., in the absence of failures. The results obtained show that the increase in the average latency experienced by messages is limited to few milliseconds.
\end{abstract}

\section{Introduction}

Computing architectures based on portable computers and wireless networking are becoming a reality. Users may be equipped with hand-held computing devices and roam around freely while maintaining connectivity with a wired computing infrastructure through a number of wireless cells.

Mobile wireless systems typically require special solutions, for a number of reasons. Traditional network protocols implicitly assume that hosts do not change their physical location over time. Mobile devices have severe resource constraints in terms of energy, processing and storage resources. Wireless networks are characterized by limited bandwidths and high error rates. Furthermore, mobility introduces new issues at the algorithmic level. For example, a mobile host may miss messages simply because of its movements, even with perfectly reliable communication links and computers that never crash [1]. All the above reasons imply that specialized protocols are required for extending to mobile hosts functionalities common for stationary ones.

In this paper we present a protocol for reliable and totally-ordered multicast within a group of mobile hosts. By this we mean that: (i) each mobile host delivers all multicasts, without duplicates; and (ii) any two mobile hosts that deliver two multicasts deliver these multicasts in the same order. 
Reliable and totally-ordered multicast is an important building block for applications composed of remote processes that have to cooperate tightly [13]. This communication primitive has proven its power in the context of traditional, i.e., static and wired, distributed computing. Our proposal makes this primitive available on mobile wireless systems. Moreover, we support this primitive in spite of (a certain number of) crashes of stationary hosts and partitions of wired links. The fault-tolerance properties of our protocol may greatly extend the scope of potential applications of mobile computing, including emergency management, plant control, traffic monitoring, stock market exchange, on-site data collection, for example. Fault-tolerant support for mobile wireless systems is, in our opinion, an important topic, yet it has not received much attention from the research community so far.

We model a mobile wireless system as follows (see figure 11. There is a set of stationary hosts (SHs) connected by a wired network and a set of mobile hosts (MHs) that may move and communicate through wireless links. Some SHs, called mobile support stations (MSSs), may communicate also through wireless links. Each MSS defines a spatially limited cell covered by a wireless link. A MSS may broadcast messages to all MHs in its cell and send messages to a specific MH in its cell, whereas a MH may only send messages to the MSS of the cell where it happens to be located. Notice that we do not assume any network support for routing messages to a specific $\mathrm{MH}$.

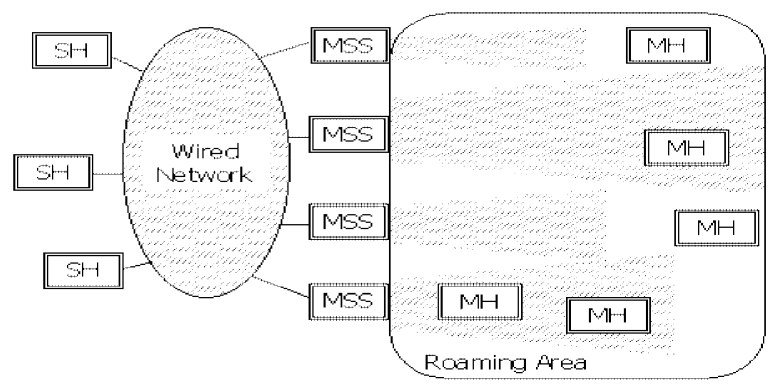

Fig. 1. Example system with five MHs and seven SHs.

An important feature of our model is the incomplete coverage of wireless cells, i.e., MHs may roam in areas that are not covered by any cell. A MH may move across adjacent cells but it may also "disappear" within the uncovered area and enter any other cell, perhaps after a "long" time. Movements occur without prior negotiation. The resulting scenario is quite general because it accommodates contemporary wireless LAN's, infra-red networks requiring line-of-sight connectivity, disconnected modes of operation, long-range movements and picocellular wireless networks in which the cell size is of the order of a few meters, such as a room in a building.

The message pattern of our protocol follows common approaches for reliable multicasting among MHs in mobile wireless systems [1,3 8,14 16]. A MH wishing to issue a multicast sends a request to the MSS of the cell where it happens to be located. The MSS forwards the message to a SH that processes this request, includes the payload, and 
forwards the payload to all MSSs. MSSs broadcast the payload in the respective cell. More details will be given later.

Our work is based on a design philosophy aimed to improve reliability of final applications, in particular, with respect to failures:

1. The state shared among SHs should not be updated upon each movement of MHs. Otherwise, performance could be penalized and failure handling would be more difficult.

2. One should avoid to assume that wireless coverage is complete. Otherwise, even a single physical obstruction, or particularly unfortunate area, or MSS malfunctioning, could compromise correctness.

3. Availability of MSSs should affect only availability of applications, not their correctness. In particular, a MSS failure should merely shrink the covered area, without affecting correctness.

4. One should avoid to make hypothesis on users' movements. Otherwise, even a single inopportune movement could compromise correctness.

5. Critical state information should not be kept on MSSs, but on "ordinary" SHs. This choice allows using systematic and established techniques for improving the availability of these hosts, such as replication.

6. MSSs should be freely added or removed without stopping the system or compromising correctness. MSS addition may be necessary for upgrading or coverage enhancement, whereas MSS removal for maintenance or failure.

Notice that the above points apply to mobile computing in general, not only to the specific problem of reliable multicast.

We have analyzed the performance of the proposed protocol by simulation. In particular, we have focused on the impact of fault-tolerance on the performance of the protocol in normal operating conditions, i.e., in the absence of failures. We have found that the proposal is indeed practical, as the latency increase due to fault-tolerance is of just a few milliseconds.

\section{System Model}

Each wired link and each wireless cell provides FIFO-ordered communication without duplicates. Messages may be lost. Message loss in a wired link occurs as a result of network partitions. Such partitions may recover. Message loss in a wireless cell may occur because of physical obstructions or because of the intrinsic features of wireless technology, e.g., high error rate. Hosts communicate solely via messages. Of course, while a $\mathrm{MH}$ is out of coverage no communication with it is possible. Similarly, SHs partitioned from each other cannot communicate among themselves. SHs may crash and a crashed SH may recover. MHs do not crash (see also below).

The system is asynchronous in the sense that neither message delays nor computing speeds can be bounded with certainty. This characterization is a general and realistic one as it allows abstracting away such features as variable loads imposed by users and unknown scheduling strategies on hosts and communication links. Notice that a process 
cannot determine with certainty whether a remote process that appears to be unresponsive has crashed or happens to be very slow.

The protocol can be easily made resilient also to crashes of MHs, the only problem being that state information about a crashed $\mathrm{MH}$ would never be discarded by SHs. A practical implementation might allow SHs to unilaterally garbage-collect state information not accessed for a very long time. Although a MH deemed crashed might show up again, there is no way to exclude such a possibility in an asynchronous system - unless one is willing to wait for an infinite time before deciding whether that $\mathrm{MH}$ actually crashed. Another practical issue is that a crashed $\mathrm{MH}$ should be able to participate again in the application after its recovery. This feature may be achieved by: (i) supporting a dynamic group of MHs that may exchange multicasts; and (ii) requiring that multicasts be delivered only by current members of the group. The protocol proposed here assumes a static set of MHs but it may be extended towards supporting (i) and (ii) quite simply [6. 9].

\section{Related Work}

To the best of our knowledge, the only work with scope similar to ours is [2]. This work introduces resilience to failures of MSSs in a non fault-tolerant reliable multicast protocol proposed by Acharya and Badrinath (see below, [1]). However the system model is much more restrictive than ours because: (i) it assumes that a process can detect with certainty whether a remote process is active or crashed (fail-stop failures); and (ii) communication is reliable, both in the wired network and in wireless cells (thereby excluding, for example, uncovered regions, physical obstructions within cells, partitions of wired links).

The protocol in [2] adds fault-tolerance to the one in [1] by associating each MH with a set of MSSs, denoted $\mathcal{S}(\mathrm{MH})$, and by replicating state information about that $\mathrm{MH}$ at each member of $\mathcal{S}(\mathrm{MH})$. Whenever $\mathrm{MH}$ sends a message or there is a message addressed to it, members of $\mathcal{S}(\mathrm{MH})$ have to execute a replica control protocol and this protocol must be able to tolerate host failures. The cited work mentions two alternatives for such protocol. The one that is more efficient requires additional mechanisms (network flush or rollback) that are not detailed. Furthermore, no performance analysis is provided and the complex interaction among (i) replica control protocol, (ii) MSS recovery, and (iii) hand-off, are only outlined 1 . Our protocol is fully detailed and, in our opinion, is much simpler to understand and implement.

The protocol by Acharya and Badrinath, hereinafter the AB-protocol, was the first multicast protocol ensuring reliable (FIFO) delivery in the context of mobile computing and has been highly influential in the design of later protocols [3,8 14 16]. Although none of these protocols is fault-tolerant, it is useful to discuss them briefly to emphasize the differences with our proposal. Each MSS maintains, for each MH in its cell, an array of sequence numbers describing the multicasts already delivered by that MH. The MSS uses this array to forward pending messages in sequence and without duplicates. If the MH switches cell, the array is moved to the new MSS by means of a proper hand-off

\footnotetext{
${ }^{1}$ The paper claims that the composition of $\mathcal{S}(\mathrm{MH})$ may change dynamically, but it appears that
} this issue has been oversimplified, in particular, with respect to the interaction just mentioned. 
procedure. Therefore: (i) The state shared among SHs is updated upon each movement; (ii) MSSs maintain critical state information (i.e., each MSS remembers the sequence numbers of multicasts delivered by each MH in its cell); and (iii) the crash of a MSS affects correctness of the application (i.e., the above sequence numbers are lost for each $\mathrm{MH}$ in the cell). These features explain why the fault-tolerant extension in [2] requires a complex interaction among several sub-protocols. The AB-protocol and the protocols derived from it assume reliable communication in the wired network and in the wireless network, much like [2].

The AB-protocol provides reliable delivery without requiring routing support for MHs, e.g., Mobile IP, much like our proposal. Multicast protocols that rely on Mobile IP are generally targeted at different application domains and provide unreliable, besteffort, unsequenced delivery [12,15]. In particular, no messages are delivered during a cell switching and messages possibly lost will not be recovered in the new cell. With respect to the use of Mobile IP, note also that: (i) it would not solve the problem of recovering from lost messages; (ii) it would make it more difficult to exploit the broadcast capabilities of the wireless medium when many MHs are in the same cell; (iii) it would generate traffic in the wired network even while no new multicasts are generated, for tracking the location of each $\mathrm{MH}$.

The protocol proposed here is an extension of the protocol in [9] that was not faulttolerant and assumed reliable communication in the wired network. As an aside, performance analysis by simulation showed that the proposal in [9] outperforms the ABprotocol in terms of latency, scalability, bandwidth usage efficiency and quickness in managing cell switches of users [4]. The proposal in [9], as well as the one in this paper, borrows a crucial idea from the implementation of reliable multicasts in "static and wired" distributed systems: the use of a centralized sequencer for totally ordering multicasts and for storing multicasts that have not been acknowledged yet [13]. Note, however, that here we refer to a completely different system model: mobile hosts, wireless communication, incomplete spatial coverage.

\section{Overview of the Protocol}

We begin by briefly outlining the non fault-tolerant version of the protocol. Messages have a field of enumerated type, called tag and indicated in SMALLCAPS, that indicates the purpose of the message. We say that a host $H$ receives a message $m$ when $m$ arrives at the protocol layer at $H$, and that $H$ delivers $m$ when the protocol forwards $m$ up to the application.

A MH wishing to issue a multicast sends the payload to the local MSS with a NEw message. $\mathrm{MH}$ retransmits this message until receiving an acknowledgment (possibly from a different MSS, if the sending MH moves during the handshake). The MSS forwards the message to a designated SH acting as coordinator. A NEw message carries a sequence number locally generated by the sending $\mathrm{MH}$, which enables the coordinator to process New messages in sequence and to discard duplicates. The coordinator constructs a NORMAL message containing the payload of the NeW message and a locally generated sequence number. The resulting message is then multicast to MSSs that broadcast it in the respective cell. Each MH uses sequence numbers of NORMAL messages to deliver 
these messages in sequence without duplicates (i.e., in total order) and to detect missing messages. In the latter case, the MH sends a retransmission request to the local MSS. This request is tagged NACK and specifies an interval of missing sequence numbers. When a MSS receives a NACK, it relays the missing NORMAL messages to the sending $\mathrm{MH}$. The MSS obtains such messages from a local cache or, in case of a miss, from the coordinator. MSS requests missing messages to the coordinator with a FETCHREQ specifying an interval of sequence numbers. The coordinator responds with a FETCHREP containing the required messages. A NACK from a MH implicitly acknowledges delivery of previous multicasts. MSSs extract this information and forward it to the coordinator, with StabInfo messages. Note that: (i) MSSs do not store critical state information: such information is kept by the coordinator and merely cached by MSSs for efficiency; (ii) each MSS reacts to cell switching without interacting with other MSSs.

The fault-tolerant extension proposed here is obtained as follows.

1. A MH no longer assumes that a message arrived at a MSS will eventually arrive at the coordinator - the MSS might crash, or a partition might occur. Instead, a MH keeps on retransmitting a NEW message until receiving the matching NORMAL message (a MSS that receives a NEW message does not respond to the sending MH with an acknowledgment, as this acknowledgment would be useless).

2. The role of the single coordinator is played by a set of SHs, called coordinators. This set appears to MSSs as a single "coordinator service". The service is available in spite of (a certain number of) failures of coordinators and connecting links. In particular, availability of the service requires a majority of coordinators. Coordinators interact among themselves through group communication $(G C)$ [10]. GC may be thought of as a software layer exporting to applications a membership service and a communication service for reliable multicasting within a group of processes. These two services are tightly integrated so as to simplify the programming of distributed algorithms in the face of host crashes and recoveries, network partitions and mergers. More details will be given in section 4.1.

3. A MSS sends its messages to a designated coordinator, say $\mathrm{C}$. The MSS might not receive a response for several reasons, including: (i) $\mathrm{C}$ is not able to interact with a majority of coordinators (section 4.1); (ii) the message from MSS to C is lost; (iii) the response from $\mathrm{C}$ to MSS is lost. Should a response not arrive within a specified timeout, the MSS will send the next request to another coordinator. The request not yet answered will be retransmitted by the originating $\mathrm{MH}$, as pointed out above (1). The policy for associating coordinators with MSSs is irrelevant to this paper. Of course, timeouts expiring too soon must not affect correctness. To this end, the coordinator service maintains internally information sufficient to detect duplicate requests (section 4.1).

Space constraints preclude a full description of the protocol, that can be found in the companion report in a pseudo-code form [5]. We will discuss in the next section only the implementation of the coordinator service.

\subsection{Coordinator Service}

Interaction among coordinators occurs through group communication $(G C)$ [10]. A detailed description of GC is beyond the scope of this paper and we provide below only 
the necessary background. GC is implemented by a dedicated software layer at each coordinator.

Coordinators form a group (this notion of group has nothing to do with the group of MHs). The GC layer provides consistent information about the set of coordinators that appear to be currently reachable. This information takes the form of views. The GC layer determines a new view as a result of crashes, recoveries, network partitions and mergers. New views are communicated to coordinators automatically, through special messages called view changes. When a coordinator $C$ receives a view change carrying the new view $V, C$ is informed that it can communicate with the coordinators listed in $V$. To proceed further, we need a few simple definitions: (i) $C$ installed a view $V$ means that $C$ indeed received the corresponding view change; (ii) two views $V, W$ are consecutive means that a coordinator installs $V$ and then installs $W$; (iii) the view that is current at $C$ is the one specified by the last view change received by $C$; (iv) $C$ delivers message $m$ in view $V$ means that $C$ delivers $m$ when the view that is current at $C$ is $V$.

The key guarantee of GC is that view changes are globally ordered with respect to the receiving of multicasts: Given two consecutive views $V$ and $W$, any two coordinators that install both views must have received the same set of multicast messages in view $V$. For example, consider a coordinator $C_{1}$ that delivered $V$ and suppose $W$ is delivered as a result of the crash of $C_{1}$. If $C_{1}$ crashed while performing a multicast $m$, then (i) all coordinators that install $V$ and $W$ receive $m$ (and do so before installing $W$ ); or (ii) none of them receives $m$. Clearly, this property is very powerful for reasoning about fault-tolerant algorithms.

The GC layer supports partitionable membership, i.e., it allows multiple views of the group to exist concurrently, to model network partitions. Moreover, the GC layer supports uniform multicast: if any member of view $V$ delivers multicast $m$, then each member of $V$ delivers $m$ or crashes. We present the algorithm in the hypothesis that a view including a majority of coordinators always exists. The algorithm may be extended to accommodate the more general case in which the majority view temporarily disappears.

The variables maintained by each coordinator include the following: boss, the identifier of a designated member of a majority view; cseq, the sequence number of the last Normal message sent; normal-buffer, a set containing all NoRMAL messages that might not be stable, i.e., that are not known to have been delivered by each $\mathrm{MH}$; finally, member-table, a table with one element for each $\mathrm{MH}$. Each element is a record whose fields are: mid, that identifies the $\mathrm{MH}$; new-num, the sequence number (generated by the $\mathrm{MH}$ ) of the last NEW message received from mid; cseq-mid, the cseq assigned to the last NoRMAL message generated upon processing a New sent by mid; delivered, the highest sequence number of a NORMAL message that has certainly been delivered by mid.

Each coordinator $C$ executes a loop in which at each iteration it receives either a message or a view change. If the current view is not a majority, $C$ skips to the next iteration $-C$ ignores all messages and waits for a sufficient number of failures to recover. Otherwise, $C$ acts as follows. Receiving of a message provokes the transmission of an AcK to the sending MSS (to prevent expiration of the time-out at MSS). In addition:

- A NEw message is forwarded to the boss. When the boss receives one such message, it multicasts the message within the majority view. Let $m$ denote a message multicast 
by the boss and let mid denote the MH that originated the associated NEW message. Upon receiving $m$, each coordinator $C$ performs the following actions: (i) extract the entry, say e-mid, of member-table associated with mid; (ii) determine whether $m$ is a duplicate and, in this case, discard $m$ without any further processing (this check is done by comparing field new-num of e-mid to the sequence number in $m$, selected by mid itself); (iii) update field new-num of e-mid; (iv) increase cseq (system-wide sequence number); (v) construct a NORMAL message $m_{N}$ including, in particular, the payload specified by mid and cseq; (vi) store a copy of $m_{N}$ in normal-buffer; finally, (vii) the boss multicasts $m_{N}$ to MSSs. In short, coordinators proceed in locksteps and, in particular, they maintain identical copies of their variables.

- A FetchReQ message is processed locally (such a message is sent by a MSS whose local cache does not contain a NoRMAL message requested by a MH). The FETCHREP reply is constructed based on the normal-buffer.

- A StabInfo message is multicast within the view (such a message describes the Normal messages certainly delivered by a specified MH). Upon receiving this multicast, each coordinator records the related information in the pertinent entry of member-table and clears from normal-buffer messages that have been delivered by every group member.

Network partitions, mergers, host crashes and recoveries are handled simply. GC reports them automatically to coordinators in the form of a new view. Upon receiving a view change, the boss sends a copy of its variables to each coordinator that was not in the previous majority view. Then, the coordinator service starts processing again messages from MSSs, as all coordinators in the new majority view have identical variables. If the boss has left the majority view (e.g., it crashed), then a new boss is elected by applying a deterministic function to the composition of the view (this function must select a member of the previous majority view). Variables to the coordinators that have possibly entered the majority view will be sent by the new boss. Notice that all coordinators receive the same view, hence they can easily coordinate their reaction to the view change based solely on the view composition, i.e., without dedicated message-exchange rounds.

It may be useful to observe what follows: (1) The boss might crash during steps (i)-(vii) above, i.e., before actually multicasting the NormaL message to MSSs. In this case, MHs will eventually detect a hole in the stream of sequence numbers and ask retransmission; (2) When surviving coordinators receive the view notifying about the crash of the boss, they will certainly have the same variables: GC ensures that prior to the view change they have delivered the same set of multicasts from the boss.

\section{Simulation}

Fault-tolerance obviously comes at a cost. A protocol designed to be fault-tolerant is likely to exhibit, even in the absence offailures, performance worse than that of a protocol that does not tolerate failures. In this section we evaluate such costs by simulation. This analysis enables us to capture the inherent cost of fault-tolerance for our proposal. Accordingly, our simulations assume reliable communication in the wired network and SHs that do not crash. The emphasis here is demonstrating that one can tolerate failures without paying excessive costs in normal conditions, i.e., in the absence of failures. 
We set the numerous parameters that characterize the protocol similar to [6], which provides a simulation analysis for the non fault-tolerant version. There are 40 cells, i.e., 40 MSSs. A MH remains in a cell for a random time interval. The length of this interval is exponentially distributed and its average $T_{\text {cell }}$ is set to 10 seconds for each $\mathrm{MH}$. Wireless coverage is complete and the message loss rate in the wireless network is $0.1 \%$. There are $100 \mathrm{MHs}$ : all of them receive multicasts $\left(N_{r}=100\right)$ whereas only 10 of them may generate 512-byte messages $\left(N_{s}=10\right)$. Message generation is a Poisson process, i.e., times between the generation of successive messages are random variables exponentially distributed. Each sender generates, on the average, 8 messages/sec corresponding to a bit rate of approximately $33 \mathrm{Kbps}$.

We consider a wireless bandwidth of $1 \mathrm{Mbps}$, in line with the bandwidth available in current Wireless LANs [7], and a wired bandwidth of $10 \mathrm{Mbps}$. Therefore, message transmission times in the wired network are one order of magnitude lower. Propagation delays, i.e., times messages take to travel from one node to another, are as follows. Wireless propagation delays are negligible as cells are supposed to be very small, (e.g., ten meters). Wired propagation delays are assumed to be exponentially distributed. The average for messages from MSSs to coordinators or back is $1.5 \mathrm{msec}$, whereas for messages from coordinators to the boss is $2 \mathrm{msec}$ (these point-to-point messages are sent through group communication). Uniform multicast amongst coordinators is modeled as an additional exponential delay with average $\left(N_{c}+0.4\right)$ msec $([11])$. We consider also processing time, i.e., time necessary to process a message. We used the same values reported in [6] as there are no substantial differences from the non fault-tolerant version.

The main metric we consider is the average message latency, i.e., the average time elapsed from the instant at which a message is generated at a sending $\mathrm{MH}$ to the instant at which the same message is delivered by a destination $\mathrm{MH}$. We analyzed latency for varying numbers of coordinators, sending MHs, mobility of MHs and message loss rate. Curves labelled as $N_{c}=1$ refer to the non fault-tolerant version while the other curves relate to the fault-tolerant protocol proposed in this paper. For the sake of space we shall focus on the differences between the two versions. The reader can refer to [6] for details about the performance of the non fault-tolerant version.

Figure 2-left shows the average latency as a function of the number of receivers for different number of coordinators $\left(N_{c}\right)$. Note that the fault-tolerant version maintains the very good scalability properties of the non fault-tolerant one. The fault-tolerant protocol exhibits higher average latency as a result of the following factors:

1. Messages experience an additional step with respect to the non fault-tolerant version: from the coordinator associated with the $\mathrm{MH}$ that originated the message to the boss.

2. When the boss receives a New message it does not multicast the related NormaL message immediately, but it sends a uniform multicast within the view and waits for delivery of this multicast.

3. New messages are implicitly acknowledged by the related NoRMAL message from the boss while in the non fault-tolerant version they are explicitly acknowledged by the local MSS. It follows that MHs have to use longer time-outs in order to minimize useless retransmissions, but this delays retransmission of messages that are actually lost.

The companion report [5] analyzes in more detail the contribution of each factor. 
Figure 2-right shows the average latency as a function of the number of senders, i.e., of the aggregate message rate. Although average latency increases with the number of senders, it is important to observe that curves for different values of $N_{c}$ are approximately parallel. In other words, the fault-tolerant version maintains approximately the same scalability properties of the non fault-tolerant one. The difference between the two protocols is due, obviously, to points 1,2 and 3 above.
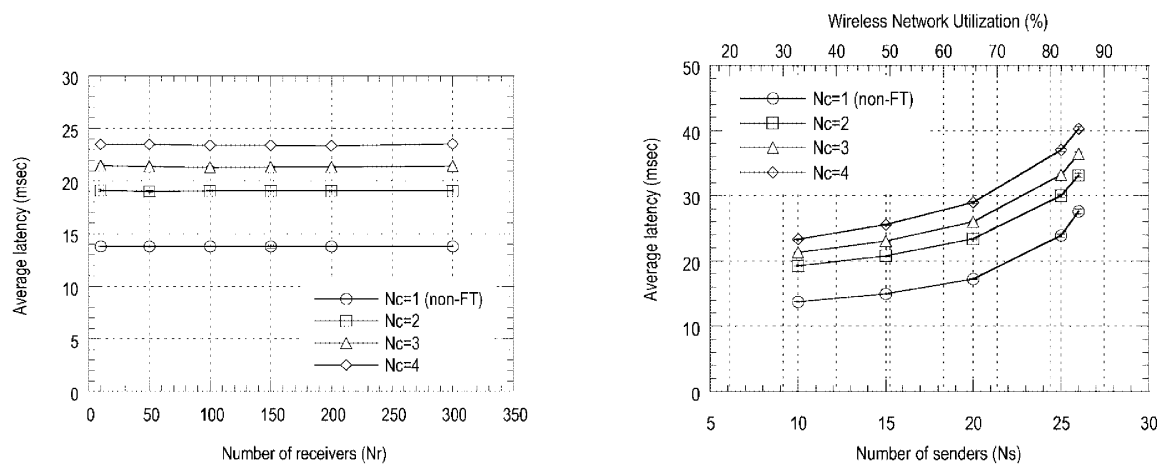

Fig. 2. Average latency as a function of the number of receivers (left) and of the number of senders (right) for different number of coordinators.
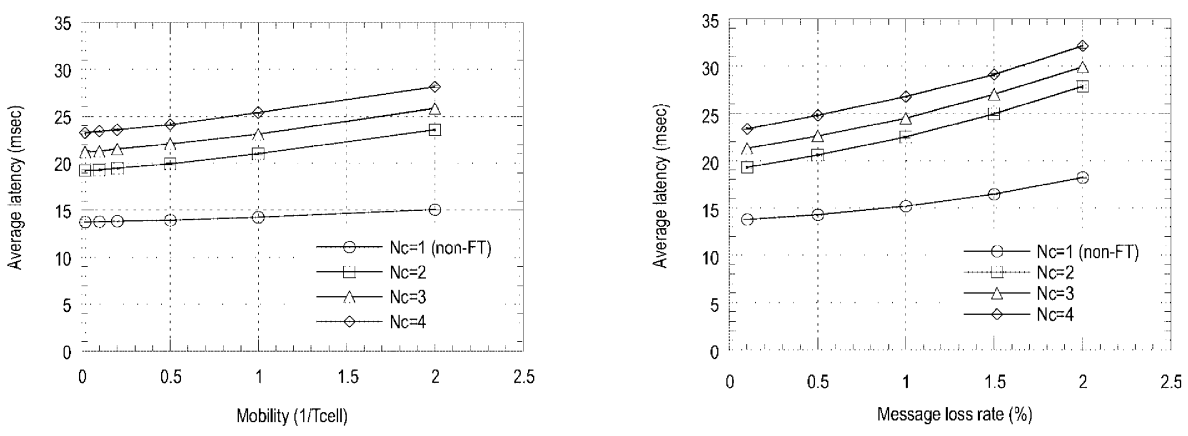

Fig. 3. Average latency as a function of mobility (left) and wireless network unreliability (right) for different number of coordinators.

Figure 3 shows the influence of $\mathrm{MH}$ mobility (left) and wireless network unreliability (right). Mobility is expressed in terms of the number of cell switches per second experienced by each $\mathrm{MH}$, which is the inverse of the $T_{\text {cell }}$ parameter, i.e., the average 
cell permanence time. Unreliability of wireless links is expressed as the percentage of lost messages.

Both plots exhibit a similar behavior. In particular, the difference between the non fault-tolerant protocol and the fault-tolerant protocol (for example with $N_{c}=2$ ) increases as either mobility or message loss rate grows up. This similarity can be easily understood if one considers that mobility of MHs may cause message losses. The increase in the distance between curves related to $N_{c}=1$ and $N_{c}>1$, respectively, is a consequence of point 3 above: when the fraction of NEW messages which get lost increases, the delay for recovering them increases accordingly.

To summarize, the latency cost induced by fault-tolerance in the absence of failures is in the order of a few msec. The above components 1 and 2 of the additional delay cannot be reduced (for a fixed wired network technology and operating environment). On the other hand, component 3 could be partially lowered by using at MHs:(i) a transmission scheme more sophisticated than the simple stop and wait approach (e.g., a windowbased scheme); and/or (ii) a shorter time-out for NEw messages. On the other hand, the former would induce higher computational load at MHs while the latter would cause useless retransmissions and, thus, wastage of wireless bandwidth as well as computing and energy resources at the $\mathrm{MH}$. Based on the above results, we believe that these solutions would lead to minor performance improvements that would not compensate for their drawbacks. However, in a different scenario, e.g., when MSSs are distributed in a geographical area rather than in a local area, use of a window-based transmission scheme could be appealing.

\section{Concluding Remarks}

We have presented a protocol for offering fault-tolerant support to (totally ordered) reliable multicast within a group of MHs. The protocol tolerates crashes of SHs and partitions of wired links. To our knowledge, no other protocol provides these functionalities.

Two key features of our protocol are: (i) movements of MHs do not require any interaction among SHs (i.e., no hand-off is required); and (ii) MSSs do not store any critical state information. Both features are crucial for coping with failures simply and efficiently. MSSs merely act as forwarding switch and as cache of state information whose primary copy is kept elsewhere, i.e., at coordinators. Replication through group communication is the main tool for enhancing availability of this information and for preserving its consistency in spite of failures.

Simulation results show that the protocol is indeed practical in that the latency cost induced by fault-tolerance in normal operating conditions, i.e., in the absence of failures, is limited to some milliseconds. Moreover the protocol exhibits very good scalability properties.

\section{References}

1. A. Acharya and B. R. Badrinath. A framework for delivering multicast messages in networks with mobile hosts. ACM/Baltzer Journal of Mobile Networks and Applications, 1(2):199-219, 1996. 
2. S. Alagar, R. Rajagoplan, and S. Venkatesan. Tolerating mobile support station failures. In Proc. of the First Conference on Fault Tolerant Systems, pages 225-231, Madras, India, December 1995. Also available as Technical Report of the University of Texas at Dallas.

3. S. Alagar and S. Venkatesan. Causal ordering in distributed mobile systems. IEEE Transactions on Computers, 46(3):353-361, March 1997.

4. G. Anastasi and A. Bartoli. On the structuring of reliable multicast protocols for mobile wireless computing. Technical Report DII/00-1, Università di Pisa and Università di Trieste, January 2000. Submitted for publication. Available at http://www.iet.unipi.it/ anastasi/papers/tr00-1.pdf.

5. G. Anastasi, A. Bartoli, and F.L. Luccio. Fault-tolerant support for reliable multicast in mobile wireless systems: Design and evaluation. Technical Report DII/02-1, Università di Pisa and Università di Trieste, March 2002. Available at http://www.iet.unipi.it/ anastasi/papers/tr021.ps.

6. G. Anastasi, A. Bartoli, and F. Spadoni. A reliable multicast protocol for distributed mobile systems: Design and evaluation. IEEE Transactions on Parallel and Distributed Systems, 12(10):1009-1022, October 2001.

7. G. Anastasi and L. Lenzini. QoS provided by the IEEE 802.11 wireless LAN to advanced data applications: a simulation analysis. ACM/Baltzer Journal on Wireless Networks, 6(2):99-108, 2000 .

8. V. Aravamudhan, K. Ratnam, and S. Rangajaran. An efficient multicast protocol for PCS networks. ACM/Baltzer Journal of Mobile Networks and Applications, 2(4):333-344, 1997.

9. A. Bartoli. Group-based multicast and dynamic membership in wireless networks with incomplete spatial coverage. ACM/Baltzer Journal on Mobile Networks and Applications, 3(2):175-188, 1998.

10. Ken Birman. The process group approach to reliable distributed computing. Communications of the ACM, 36(12):37-53, December 1993.

11. R. K. Budhia. Performance engineering of group communication protocols. Technical report, University of California, S. Barbara (USA), August 1997. Ph.D. dissertation.

12. V. Chikarmane, C. Williamson, R. Bunt, and W. Mackrell. Multicast support for mobile hosts using mobile IP: Design issues and proposed architecture. ACM/Baltzer Journal of Mobile Networks and Applications, 3(4):365-379, 1998.

13. F. Kaashoek and A. Tanenbaum. An evaluation of the Amoeba group communication system. In Proc. 16-th IEEE-ICDCS, pages 436-447, May 1996.

14. R. Prakash, M. Raynal, and M. Singhal. An efficient causal ordering algorithm for mobile computing environments. Journal of Parallel and Distributed Computing, March 1997.

15. G. Xylomenos and G. Polyzos. IP multicast for mobile hosts. IEEE Personal Communications, pages 54-58, January 1997.

16. L. Yen, T. Huang, and S. Hwang. A protocol for causally ordered message delivery in mobile computing systems. ACM/Baltzer Journal of Mobile Networks and Applications, 2(4):365$372,1997$. 